\title{
On the Application of Topology Optimisation Techniques to Thermal Management of Microelectronics systems
}

\author{
M. Santhanakrishnan*, T. Tilford, C. Bailey \\ The University of Greenwich, Park Row, London UK \\ m.santhanakrishnan@greenwich.ac.uk
}

\begin{abstract}
In this paper, an autonomous thermal management design process based on a topological optimisation algorithm is presented. The numerical framework uses a finite element multiphysics solver to assess fluid flow and heat transfer, coupled with the Method of Moving Asymptotes approach for topology optimisation. The design framework is utilised to develop a copper heatsink for a simplified electronics package at two differing Reynolds numbers. In both cases, the final shape resembles a tree like structure rather than a more conventional fin structure.
\end{abstract}

\section{Introduction}

One of the key challenges in development of modern microelectronics systems is thermal management. This is becoming an increasingly critical area of microelectronics packaging design due to the ever increasing levels of miniaturisation, integration and operating frequency which result in significantly higher power densities [1]. These thermal design challenges need to be evaluated in terms of a holistic co-design framework capable of balancing differing aspects of the design (thermal, structural, electrical etc.) to develop an overall optimum design.

Development of an electronics package through adoption of a co-design framework will involve a high level of autonomous design, wherein numerical algorithms are used not only to assess physical phenomena - for example, using FEM analysis to determine structural deformations under thermal load but to assess the overall performance of a design and to develop modifications to the design intended to improve performance. The author's view is that topological optimisation approaches are able to fulfil the requirement to autonomously modify the design of a component in order to enhance its performance.

Topology optimisation is a mathematical approach that optimises material layout within a given design space, for a given set of constraints such that the resulting layout meets a prescribed set of performance objectives. Using topology optimisation, engineers can find the best design concept to address performance requirements.
Currently topological optimisation techniques are predominantly applied in the structural mechanics field. However, the use of these techniques is now becoming increasingly adopted in a diverse range of areas. Topology optimisation algorithms typically involve three major steps namely, solving the structural/ fluid flow physics problem, evaluating the sensitivity of the objective with respect to design variable and solving the optimisation problem. These steps advance the design from and initial condition to an enhanced design. These steps are repeated in an iterative manner, advancing to a final optimal design [2].

Borrvall and Petersson [4] pioneered the use of topology optimisation of fluid flow problems namely to Stokes flow. Olesen et.al [5] extended the study to Navier Stoke flows using the FEMLAB Finite Element Method software for solving the governing equations and sensitivity calculation. Fluid flow is modelled through porous fluid flow (Brinkman approach) in which the material impermeability is modelled as a function of design variable. Both these papers used the Solid Isotropic Material with Penalization (SIMP) and Method of Moving Asymptotes (MMA) approaches to optimise the fluid flow problems.

Bruns [6] extending the use of topology optimisation to multi-physics (heat transfer and fluid flow) problems through study of forced convection heat transfer problems and the associated numerical instabilities. This work led to development of a number of algorithm stabilisation approaches. Dede [7] studied the design of a three terminal heat transfer and fluid flow device through topology optimisation using the COMSOL multiphysics package combined with a MMA solver. A dual objective function was used to minimise the mean temperature and total fluid power dissipated. The solid region created in the optimisation has zero thermal conductivity and minimum porosity.

Yoon [8] carried out the design of a heat dissipating structure subjected to forced convection and for the first time he interpolated thermal conductivity and other relevant material properties with respect to spatially defined design variables. Thereby the resulting solid regions had the non-zero thermal conductivity. Lee [9] optimised the design of a flow channel to achieve 
maximum cooling efficiency, ensuring different (and nonzero) thermal conductivities for fluid and solid regions. Koga [10] carried out the topological optimisation and experimental testing of a 3 terminal device similar to [7] but using water as the fluid and aluminium as the solid. Alexandersen [11] has carried out topology optimisation for forced convection heat transfer problems with interpolation of thermal conductivity. However, instead of directly interpolating the material properties an interpolation function for Peclet number is utilised.

\section{Microelectronics heatsink design}

This study has considered a simplistic geometry as a starting point and is limited to analyses in either two planar dimension or to axisymmetric cases. The approach is very much applicable to three dimensions which will form future work.

In the analyses presented, a rectangular copper block is placed in a forced flow of air and is subjected to a constant heat flux through a section of its lower face. The optimisation algorithm is used to determine the regions of the block which should be removed (or should become fluid region) in order to increase the heat flux from the block. The heat transfer and fluid flow problems are solved using the COMSOL multiphysics package which utilises the finite element method. The topological optimisation approach utilises the MMA solver developed by Svanberg [3], which is available in COMSOL.

\section{Numerical Model}

The first step of the optimisation process is to determine an assessment of the quality of the design. In this study, the aim is to minimise the thermal compliance of the heatsink, requiring an evaluation of fluid flow and heat transfer within the domain. This has been achieved through use of the COMSOL multiphysics package. Topological optimisation approaches typically define a design variable, $\gamma$, which lies in the range 0 to 1 . A $\gamma$ value of 1 in a finite element cell defines it as a solid and a $\gamma$ value of zero defines the cell as containing fluid. In pure heat conduction problems the thermal conductivity is modelled as a function of $\gamma$ [2]. For topology optimisation of fluid flow problems flow impermeability term is modelled as a function of $\gamma$ based on the Brinkman approach. The governing equations for the fluid flow and heat transfer are:

$$
\begin{gathered}
(\nabla \cdot u)=0 \\
\rho(u \cdot \nabla u)=-\nabla p+\nabla \cdot\left\{\mu\left\{\nabla u+(\nabla u)^{T}\right\}\right\}-\alpha u \\
\rho C p(u \cdot \nabla T)=\nabla \cdot(k \nabla T)+Q
\end{gathered}
$$

Equation 1 is the continuity equation (for steady, incompressible flow) with $\mathrm{u}$ being the velocity vector, The momentum conservation equation is given in equation 2 , relating $\mathrm{u}$ to pressure, $\mathrm{p}$, density $\rho$ and an additional term which considers the porosity effect as an additional force term. The material impermeability term, $\alpha$ is expressed in terms of design variable gamma [8] as follows.

$$
\alpha(\gamma)=\alpha_{\max } \gamma^{3}
$$

The thermal conductivity, specific heat capacity and density are also interpolated with respect to the design variable gamma in the design domain as follows.

\begin{tabular}{|c|c|}
\hline Name & Expression \\
\hline$K(\gamma)$ & $\left(\mathrm{K}_{\mathrm{s}}-\mathrm{K}_{\mathrm{f}}\right) * \gamma^{3}+\mathrm{K}_{\mathrm{f}}$ \\
\hline $\mathrm{Cp}(\gamma)$ & $\left(\mathrm{Cp}_{\mathrm{s}}-\mathrm{Cp}_{\mathrm{f}}\right) * \gamma^{3}+\mathrm{C} \mathrm{p}_{\mathrm{f}}$ \\
\hline$\rho(\gamma)$ & $\left(\rho_{\mathrm{s}}-\rho_{\mathrm{f}}\right) * \gamma^{3}+\rho_{\mathrm{f}}$ \\
\hline
\end{tabular}

Table I: Material property values

This ensures that when gamma is 1 , the parameters are equal to solid material properties and when gamma is zero, these are equal to fluid material properties. The power factor of 3 has been used to penalise intermediate values of gamma.

Once the COMSOL software has determined the thermal compliance for a given design, the topological optimisation algorithm is used to determine a modification intended to enhance the performance of the design which, in this case, relates to a reduced thermal compliance. This study had utilised the Method of Moving Asymptotes (MMA) algorithm of Svanberg [3]. This method is an improvement over Convex Linearization, such that the degree of conservatism can be controlled to achieve better convergence of optimisation problems. This approach has been widely used in topology optimisation of structural problems. In this the given objective $\&$ constraint functions are approximated as:

$$
g_{i}^{k}(\gamma)=r_{i}^{k}+\sum_{j=1}^{n}\left(\frac{p_{i j}^{k}}{U_{j}^{k}-\gamma_{j}}-\frac{q_{i j}^{k}}{\gamma_{j}-L_{j}^{k}}\right)
$$

where

$$
p_{i j=}^{k}=\left\{\begin{array}{cl}
\left(U_{j}^{k}-\gamma_{j}^{k}\right)^{2} \frac{\partial g_{i}\left(\gamma^{k}\right)}{\partial \gamma_{j}} & \text { if } \frac{\partial g_{i}\left(\gamma^{k}\right)}{\partial \gamma_{j}}>0 \\
0 & \text { Otherwise }
\end{array}\right.
$$

$$
q_{i j=}^{k}\left\{\begin{array}{lr}
0 & \text { if } \frac{\partial g_{i}\left(\gamma^{k}\right)}{\partial \gamma_{j}} \geq 0 \\
-\left(\gamma_{j}^{k}-L_{j}^{k}\right)^{2} \frac{\partial g_{i}\left(\gamma^{k}\right)}{\partial \gamma_{j}} & \text { Otherwise }
\end{array}\right.
$$




$$
r_{i}^{k}=g_{i}\left(\gamma^{k}\right)-\sum_{j=1}^{n}\left(\frac{p_{i j}^{k}}{U_{j}^{k}-\gamma_{j}}-\frac{q_{i j}^{k}}{\gamma_{j}-L_{j}^{k}}\right)
$$

The term $g_{0}$ indicates the objective function and $g_{1}, g_{2}$ etc are constraints. Subscript ' $j$ ' stands for the element number of the design domain and superscript ' $k$ ' stands for iteration number. $L_{j}$ and $U_{j}$ are moving asymptotes that are changed during the iterations such that $\gamma_{j}$ is always bounded between $L_{j}$ and $U_{j}$. Suitably changing the values of $L_{j}$ and $U_{j}$ changes the level of conservatism and rate of convergence. This convex separable problem can be solved using Lagrangian duality. In broader terms, the sequence of sub problems are solved according to the following iterative scheme:

STEP (0) A starting point $\gamma^{(0)}$ is chosen for iteration $\mathrm{k}=0$

STEP (I) For a given iteration, $k$, the following are determined:

(i) Constraint function value: $\mathrm{g}_{1}\left(\gamma^{(\mathrm{k})}\right)$

(ii) Gradients (in terms of $\gamma$ ) of the cost function as well as the constraint functions: $\nabla g_{0}\left(\gamma^{(\mathrm{k})}\right)$ and $\nabla g_{1}\left(\gamma^{(\mathrm{k})}\right)$

STEP (II) Generate a sub-problem based on the original problem by replacing the original implicit functions with approximating explicit functions based on the results of STEP (I).

STEP (III) Find the optimal solution of the sub-problem and let this solution be the next iteration point $\gamma^{(\mathrm{k}+1)}$. Go to STEP (I) and repeat until some convergence criterion is met.

From the lower bound and upper bound of the design variables, moving asymptotes $\mathrm{U}_{\mathrm{j}}$ and $\mathrm{L}_{\mathrm{j}}$ values can be calculated for any problem as:

$$
\begin{aligned}
& L_{j}^{k}=\gamma_{j}^{k}-s_{\text {init }}\left(\gamma_{j}^{\max }-\gamma_{j}^{\text {min }}\right) \\
& U_{j}^{k}=\gamma_{j}^{k}+s_{\text {init }}\left(\gamma_{j}^{\text {max }}-\gamma_{j}^{\text {min }}\right)
\end{aligned}
$$

Where $s_{\text {init }}$ is a fixed real number. The above expression or ' $s$ ' value can be altered depending on the nature of solution progress. That is the expression can be suitably chosen to prevent any oscillation in the solution as well as slow convergence and monotonicity. This method works excellently for structural optimisation problems.
As previously stated, the objective of the optimisation process is to minimise thermal compliance, yielding minimum average temperature over the entire design domain. The Reynolds number ( $\mathrm{Re}$ ) plays a significant role in choosing the value of $\alpha$; If $\mathrm{Re}$ is low, higher values of $\alpha$ can be used thereby solid can be accurately modelled but if $\mathrm{Re}$ is comparatively large higher $\alpha$ values possess problem in flow start and convergence. Physically this could be explained as at low Reynolds number flow diffuses in to the porous media more easily than at high Reynolds number.

\section{Problem Definition}

The 2D computational domain used for this study is shown in Figure1. The design domain is rectangular in shape and at its base a $5 \mathrm{~mm}$ thick section of strip of solid material representing a microelectronics heat source is heated by a heat flux of $100 \mathrm{~W} / \mathrm{m}^{2}$. The top surface of the computational domain is defined as a fluid inlet with a prescribed constant velocity matching the Reynolds number of the study. The two vertical sides are fluid outlets. The bottom side (excluding the base of the design domain) is considered to be an adiabatic no slip wall.

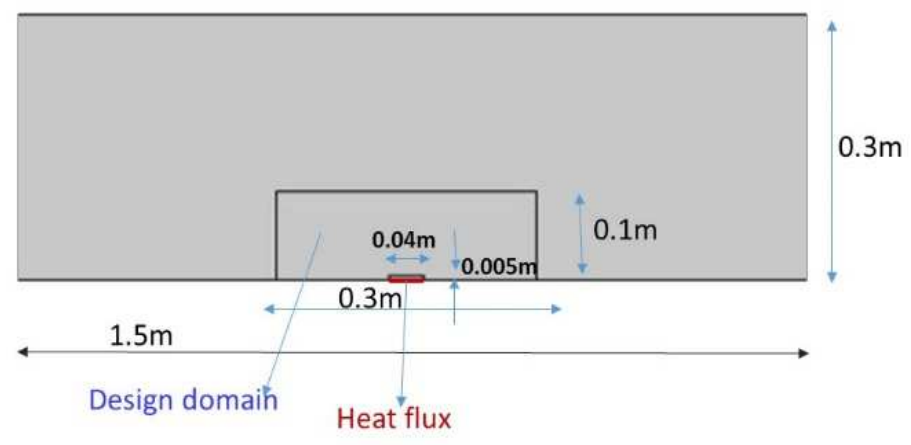

Figure 1: Computational domain details

For these types of problem, the thermal conductivity ratio between fluid and solid and Reynolds number of the flow are the most influential parameters. The materials used in this particular study are copper and air, resulting in a conductivity ratio of $1.5603 \mathrm{e}+4$. The influence of Reynolds number on the optimal design is studied by conducting the simulations at $\mathrm{Re}=12$ and $\mathrm{Re}=70$ corresponding to velocities $6.171 \mathrm{e}-4$ and $3.6 \mathrm{e}-3 \mathrm{~m} / \mathrm{s}$ respectively. The design domain length is considered as the characteristics length $(0.3 \mathrm{~m})$. The parameter values used in the different simulations are tabulated below.

\begin{tabular}{|c|c|}
\hline Parameter & Value \\
\hline$\alpha_{\max }$ & $1 \mathrm{e} 6$ \\
\hline $\mathrm{K}_{\mathrm{s}} / \mathrm{K}_{\mathrm{f}}$ & 15603 \\
\hline$\rho_{\mathrm{s}} / \rho_{\mathrm{f}}$ & $8920 / 1.225$ \\
\hline $\mathrm{Cp}_{\mathrm{s}} / \mathrm{Cp}_{\mathrm{f}}$ & $385 / 1005$ \\
\hline
\end{tabular}

Table II: Analysis parameter values 
The volume fraction of solid material is constrained to 0.4. The domain is discretised with triangular cells using a Delaunay triangulation method. Typically meshes with in the order of 50,000 elements were used, with refinement used to concentrate cells within the design section of the computational domain.

The approach adopted in this work can be prone to numerical stability and convergence issues. In order to attempt to mitigate against these, spatial discretisation is limited through imposition of equation 11 and through initialisation of the higher Reynolds number case with the optimal solution obtained in the lower Reynolds number case. The global objective value in the design domain is also monitored. Typically in a converged run the relative change in control variable gamma is less than $1 \mathrm{e}-5$.

$$
\left(\frac{\delta y^{2}}{\partial x}\right)+\left(\frac{\delta y^{2}}{\partial y}\right) \leq 150
$$

\section{Two Dimensional simulations}

The topological optimisation framework is an iterative process with the heatsink shape and resulting thermal compliance evolving during the solution process. Figure 2 shows the evolution of solid material for the two dimensional simulation at $\operatorname{Re}=12$. The iterative process is continued until changes in the global objective value decrease below a critical value, defined as $1 \mathrm{e}-3$ in this study.

Convergence of global objective function is shown in Figure 3. Figure 4 shows the optimised shape (solid material layout) for the $\mathrm{Re}=12$ case, while results for the $\mathrm{Re}=70$ case are shown in Figure 5. The optimal solid material layout resembles a tree-like structure, with the main branch leading towards the corner of the design domain and other secondary branches extending towards the edges of the design domain. The optimal tree shape is in agreement with the constructal theory of Bejan [12], wherein he states that a system will evolve in such a way that it provides easier access to the imposed currents that flow through it. Near the edge of the design domain the branch expands like a fan covering almost entire length of domain edge with solid material. This could be to effectively enhance the convective cooling happening by the downward air stream.

The global objective value for analyses at both Reynolds numbers are of same order, with results given in table III . Simulations with different gamma initialisation lead to different optimal values and the given result had the minimum compliance among all the runs. The results show that values of the design variable $\gamma$ lies between zero and unity in a number of cells, leading to a blurring of the interface. This intermediate region is one of the drawbacks of the SIMP methodology. Work to overcome this through adoption of a Level-Set approach [13] is being performed.

\begin{tabular}{|c|c|}
\hline Case & Global objective $\mathrm{kgmK} / \mathrm{s}^{3}$ \\
\hline $\operatorname{Re} 12$ & 0.0933 \\
\hline $\operatorname{Re} 70$ & 0.0961 \\
\hline
\end{tabular}

Table III: Thermal compliance results

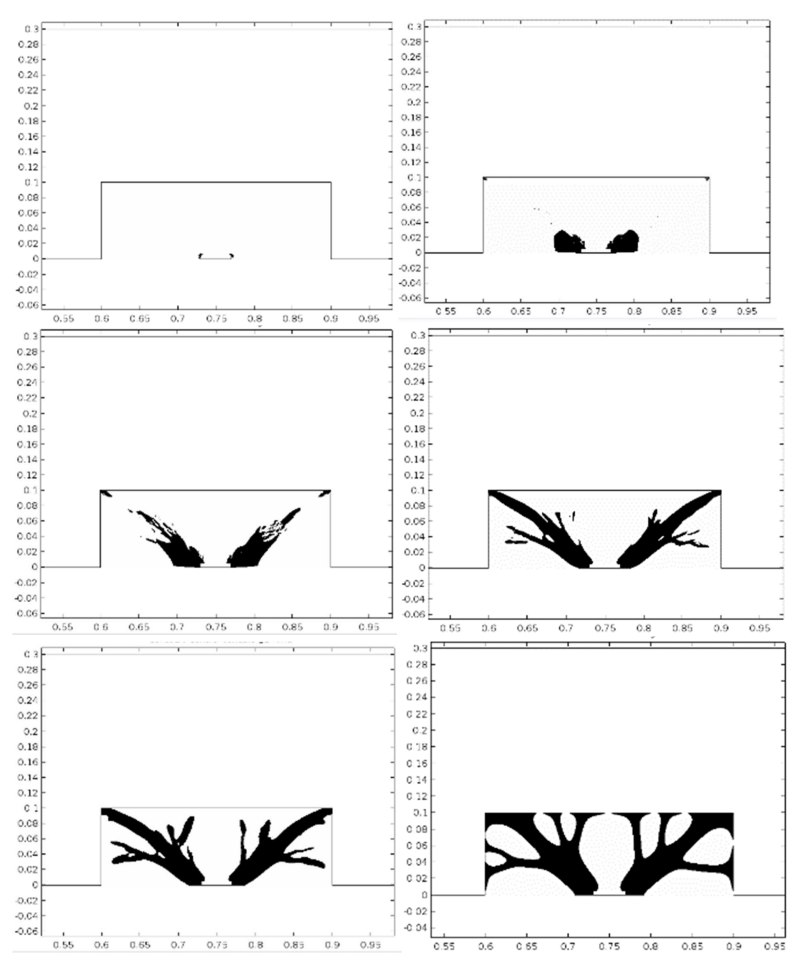

Figure 2: Evolution of solid material distribution during optimisation process

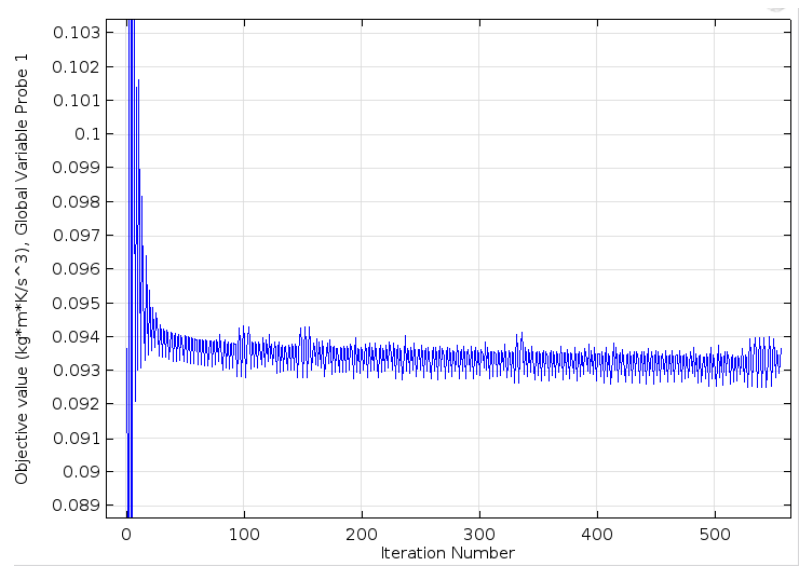

Figure 3: Convergence plot of objective function 

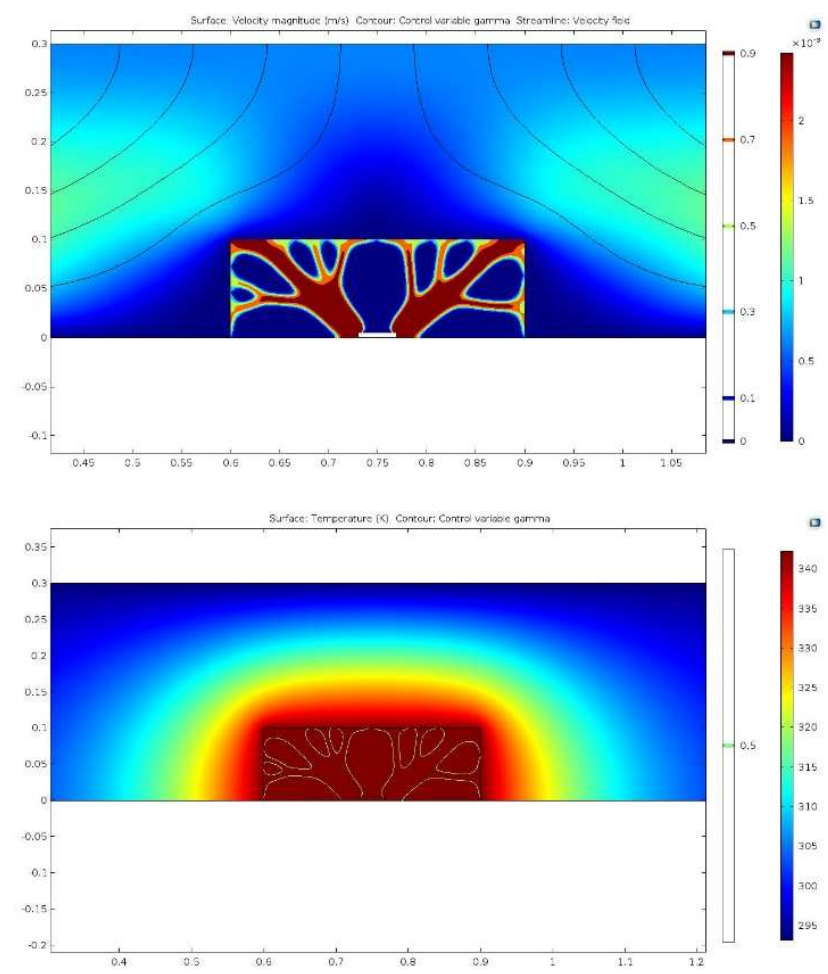

Figure 4: Optimised solid material layout with velocity contour (top) and temperature contours (bottom) for $\mathrm{Re}=12$
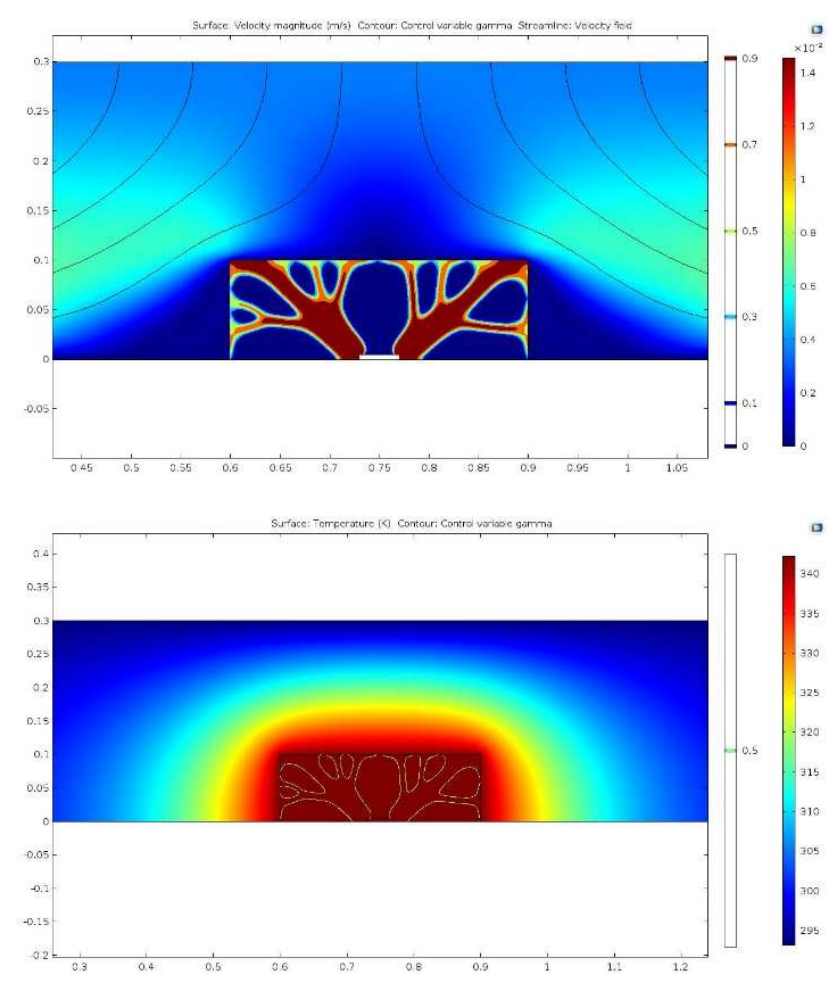

Figure 5: Optimised solid material layout with velocity (top) and temperature contour (bottom) for $\mathrm{Re}=70$

\section{Axisymmetric simulations}

In this study topology optimisation technique is used to design an axisymmetric heat sink subjected to forced convection. As practical adoption of an axisymmetric heatsink is unlikely in practice, this study is carried out for academic interest. Half of the 2D computational domain is considered for the study with axial symmetry condition on the left side surface. A higher surface heat flux of $1000 \mathrm{~W} / \mathrm{m}^{2}$ is specified in this study $\left(100 \mathrm{~W} / \mathrm{m}^{2}\right.$ is specified in 2D study). Rest of the boundary conditions and mesh are kept similar to the 2D case.

The optimised shape with velocity contour and a 3D iso-surface for $\mathrm{Re}=12$ and $\mathrm{Re}=70$ are shown in Figure 6 and 7 respectively. The $\mathrm{Re}=70$ needed to be initialized with the $R e=12$ case to avoid numerical instability issues. The optimal design formed in the axisymmetric cases is broadly similar to the two dimensional results. However, the number of branches in the axisymmetric case is comparatively higher than the $2 \mathrm{D}$ case, with the base of the tree also being much thicker.
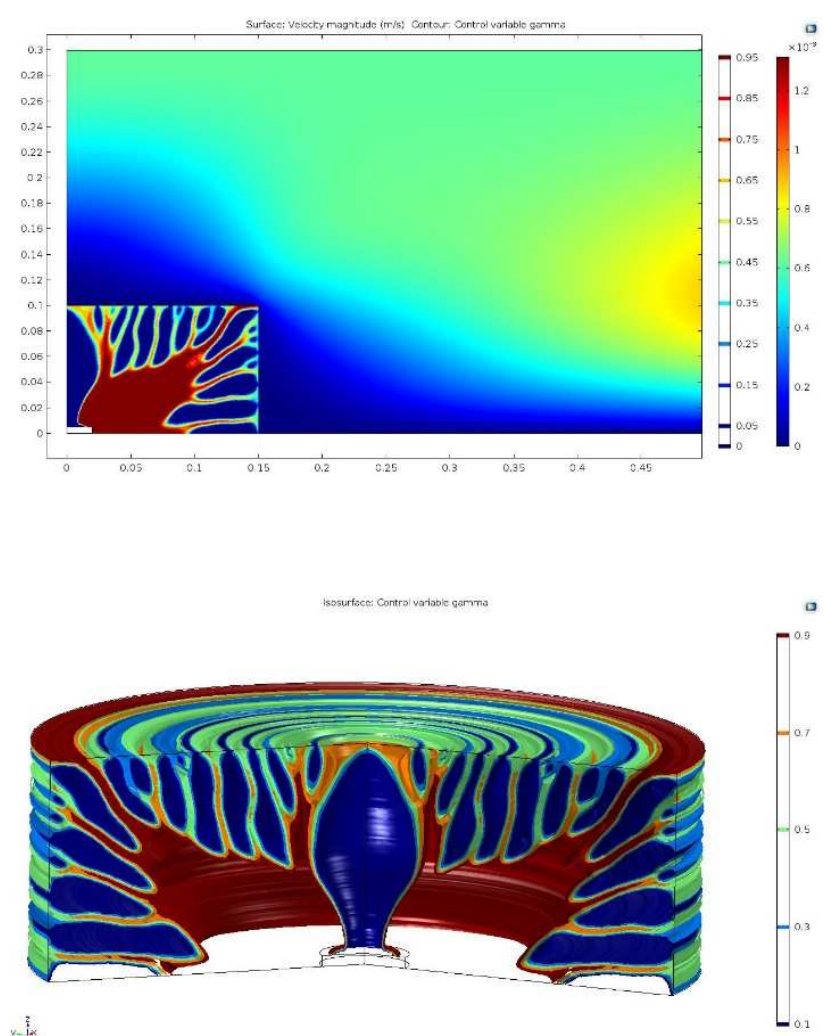

Figure 6: Optimised solid layout, velocity contour (top) and axi-symmetric solid layout (bottom) at $\mathrm{Re}=12$ 

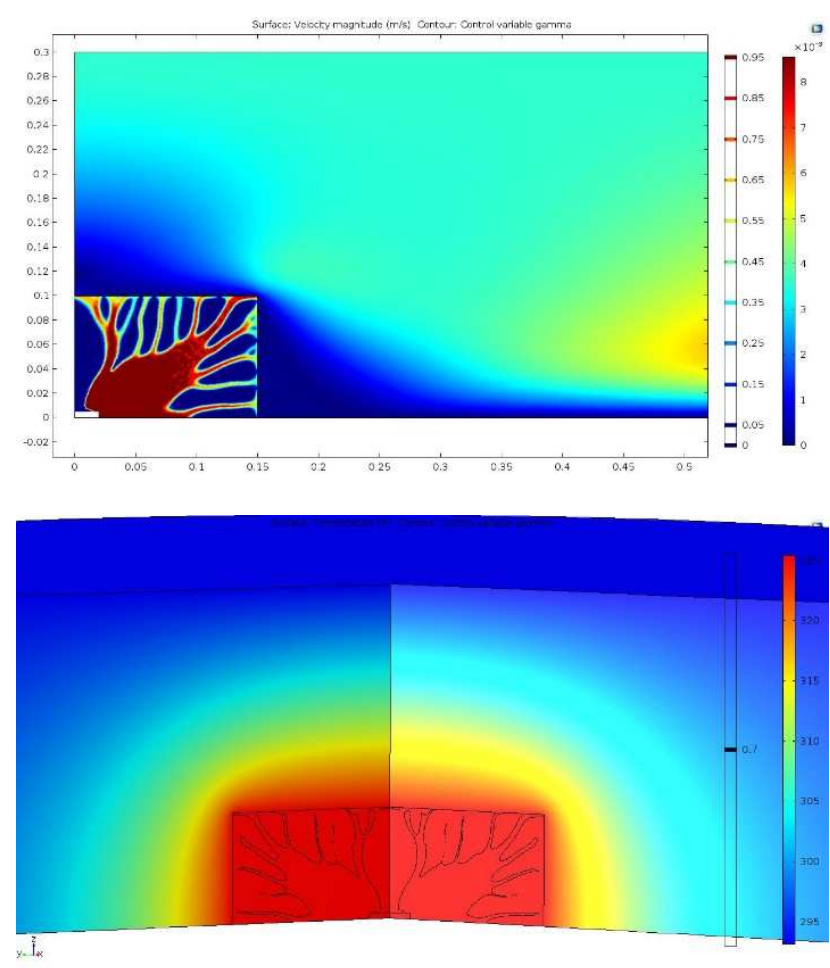

Figure 7: Optimised solid layout, velocity contour (top) and temperature contour (bottom) at $\mathrm{Re}=70$

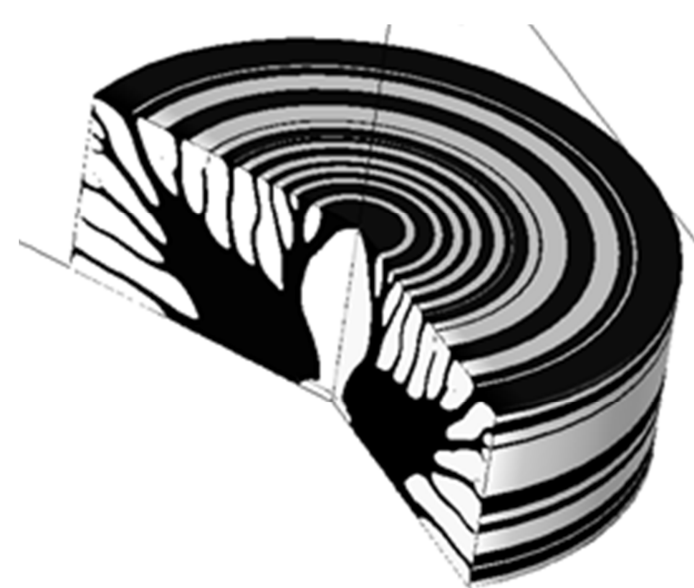

Figure 8: Optimised solid material shape at $\operatorname{Re} 70$

The optimal solid material shape is shown in Figure 8 . The global objective value obtained for different cases are tabulated below. Objective values did not vary with the tested Reynolds numbers, may be because the Reynolds numbers are not very different from one another.

\begin{tabular}{|c|c|}
\hline & Global objective $\left(\mathrm{kgmK} / \mathrm{s}^{3}\right)$ \\
\hline $\operatorname{Re} 12$ & 0.27498 \\
\hline $\operatorname{Re} 70$ & 0.27473 \\
\hline
\end{tabular}

Table IV: Thermal compliance results

\section{Conclusions}

The design of a copper heat sink subjected to forced convective cooling by air has been demonstrated. The methodology is based on a finite element multiphysics analysis solver together with the Globally Convergent Method of Moving Asymptotes (GCMMA) of Svanberg. The heatsink design problem was investigated at two different Reynolds numbers. The optimal heatsink shapes resemble tree-like forms as expected. It was found that the Reynolds number variations considered in this paper do not play a significant role on the shape of solid layout. The optimal shape of the full axisymmetric case also resembles a tree but here the number of branches are higher than the planar case.

\section{Acknowledgements}

Authors wish to acknowledge Dr..Kristian Ejlebjærg Jensen, Nanotechnology division, Denmark Technical University (DTU) for the support he rendered during the course of this work. The author would also like to express financial support from the University of Greenwich for sponsoring this work as part of a $\mathrm{PhD}$ study.

\section{References}

1. R. R. Tummala, "Packaging: Past, Present and Future", 6th International Conference on Electronic Packaging Technology, 2005, Shenzhen, China

2. M. P. Bendsøe, O. Sigmund, Topology Optimization: Theory, Methods and Applications, Springer, second edition, 2004.

3. Svanberg, K., "Method of Moving Asymptotes - a New Method for Structural Optimization", International Journal for Numerical Methods in Engineering, volume 24, no. 2, February 1987, pp. 359-373.

4. T. Borrvall, J. Petersson, "Topology optimization of fluids in stokes flow", International Journal for Numerical Methods in Fluids Vol 41, 2003, pp. 77107.

5. Olesen LH, Okkels F, Bruus H. "A high-level programming-language implementation of topology optimization applied to steady-state Navier-Stokes flow", International Journal for Numerical Methods in Engineering, Vol 65, No 7, 2006, pp. 957-1001.

6. T.E. Bruns, "Topology optimization of convectiondominated, steady-state heat transfer problems", International Journal of Heat \& Mass Transfer, Vol 50, (2007) pp. 2859-2873.

7. E.M. Dede, "Multiphysics topology optimization of heat transfer and fluid flow systems", Proceedings of the COMSOL Conference, Boston, 2009.

8. Yoon GH. "Topological design of heat dissipating structure with forced convective heat transfer". 
Journal of Mechanical Science and Technology, Vol 24, No 6, 2010, pp. 1225-1233.

9. Lee K. Topology optimization of convective cooling system designs. PhD Thesis, University of Michigan 2012.

10. Koga AA, Lopes ECC, Nova HFV, de Lima CR, Silva ECN, "Development of heat sink device by using topology optimization", International Journal of Heat and Mass Transfer, Vol 64, 2013, pp. 759-772.

11. Alexandersen J, "Topology optimisation for coupled convection problems" Master's Thesis, Technical University of Denmark 2013.

12. Bejan, A. "Constructal-theory network of conducting paths for cooling a heat generating volume", International Journal of Heat and Mass Transfer, Vol 40, 1996, pp. 799-816

13. Peter Coffin, Kurt Maute, "Level set topology optimization of cooling and heating devices using a simplified convection model", Structural and multidisciplinary optimization, December 2015 